OPEN ACCESS

Edited by:

Alessandro Granito, University of Bologna, Italy

Reviewed by:

Piero Portincasa

University of Bari Aldo Moro, Italy Linda Beenet, University of California, Los Angeles,

United States

*Correspondence:

Dawei Shi

shidawei800@126.com orcid.org/0000-0002-4141-976X

tThese authors have contributed equally to this work and share first authorship

Specialty section: This article was submitted to Gastroenterology, a section of the journa

Frontiers in Medicine

Received: 31 December 2020 Accepted: 25 October 2021

Published: 15 November 2021

Citation:

Zeng Y, Dai Y, Zhou Z, Yu X and Shi D (2021) Hepatotoxicity-Related

Adverse Effects of Proton Pump Inhibitors: A Cross-Sectional Study of Signal Mining and Analysis of the FDA

Adverse Event Report System Database. Front. Med. 8:648164. doi: 10.3389/fmed.2021.648164

\section{Hepatotoxicity-Related Adverse Effects of Proton Pump Inhibitors: A Cross-Sectional Study of Signal Mining and Analysis of the FDA Adverse Event Report System Database}

\author{
Yifan Zeng ${ }^{1 \dagger}$, Ying Dai ${ }^{2 \dagger}$, Ziye Zhou ${ }^{2}$, Xuben $\mathrm{Yu}^{2}$ and Dawei Shi ${ }^{2 *}$ \\ ${ }^{1}$ Computer Technology and Information Centre, First Affiliated Hospital of Wenzhou Medical University, Wenzhou, China, \\ ${ }^{2}$ Department of Pharmacy, First Affiliated Hospital of Wenzhou Medical University, Wenzhou, China
}

Background and Objectives: Mounting evidence demonstrates that proton pump inhibitors (PPIs) are associated with a number of adverse effects. However, the literatures about hepatotoxicity-related adverse effects (HRAEs) of PPIs are mostly case reports and a few clinical studies.

Methods: We evaluated the association between PPIs and HAREs using the reporting odd ratio $(\mathrm{ROR})$ for mining the adverse event report signals in the FDA Adverse Event Reporting System (FAERS) database.

Results: There were 23,825 reports of PPls as primary suspect drug or second suspect drug, of which 3,253 reports were HRAEs. The top five HRAE signals caused by PPIs were hepatitis cholestatic, cholestasis, fulminant hepatitis, subacute hepatic failure, and acute hepatitis. We also summarized the signals of the HRAEs caused by each PPI. The simultaneous signals were cholestasis and hepatitis cholestatic. For the cholestasis signal, esomeprazole showed an ROR of 21.556 (95\% Cl 17.592-26.413); pantoprazole showed the highest ROR of 22.611 (95\% Cl 17.794-28.733) in the hepatic cholestatic signal; lansoprazole was the only PPI with expression in the coma hepatic signal, with an ROR of 10.424 (95\% Cl 3.340-32.532). By analyzing the reports of pantoprazole-induced hepatic encephalopathy, we found that patients aged over 65 years and males reported the highest rate. And from the combination of drugs and indications of drugs, no significant results were obtained.

Conclusions: The RORs of signals of "cholestasis" were generally higher than those of "hepatocellular injury." And the signals about "cholestasis" in HRAE caused by PPIs are more reported.

Keywords: proton pump inhibitors, hepatotoxicity, cholestasis, coma hepatic, hepatitis fulminant 


\section{INTRODUCTION}

Proton pump inhibitors (PPIs) are a class of medications that work to decrease gastric acid and are FDA-approved for the treatment of a variety of acid-related conditions, including duodenal ulcers, gastric ulcers, erosive esophagitis, gastroesophageal reflux disorder, Helicobacter pylori eradication, and pathological hypersecretory conditions (1). Since the introduction of omeprazole (OME) in 1989, PPIs have demonstrated consistent patient tolerance and excellent safety compared with prior agents (2). Recently, mounting evidence has demonstrated that PPIs are related to a number of adverse effects, including acute and chronic kidney disease, hypomagnesemia, Clostridium difficile infection, pneumonia, and osteoporotic fractures, and so on (3). However, the literature about hepatotoxicity-related adverse effects (HRAEs) of PPIs are mostly case reports and a few large clinical studies (4-6). We also found in VigiAccess of the Uppsala Monitoring Center that HRAEs caused by PPIs are not common (7). Taking Omeprazole as an example, among the 108,954 cases reported, only 2,156 reports from the hepatobiliary system were reported, accounting for only $2.0 \%$ of the reports. Therefore, physicians tend to ignore the HRAEs caused by PPIs in clinical practice.

The FDA Adverse Event Reporting System (FAERS) includes several million spontaneous reports of drug-associated adverse events and is used to evaluate drug safety profiles $(8,9)$. It is one of the primary tools used for post-marketing surveillance and pharmacovigilance because it is the largest, most well-known database worldwide, and it reflects the realities of clinical practice.

In our study, adverse event reports submitted to the FDA were reviewed to assess the adverse event profiles of 7 PPIs: omeprazole, esomeprazole, lansoprazole, dexlansoprazole, rabeprazole, pantoprazole, and ilaprazole. Data mining algorithms were used for the quantitative detection of signals, in which a signal represents an association between a drug and an adverse event $(10,11)$. The adverse events related to hepatotoxicity were analyzed. To our knowledge, our study was the first to evaluate the association between PPIs and HRAEs using the reporting odds ratio (ROR) for mining the adverse event report signals in the FAERS database (12).

\section{METHODS}

\section{Data Source}

The study was designed as a retrospective study, and adverse drug event quarterly report files from Jan 2013 to Dec 2019 in the FAERS database were downloaded from the FDA website (13). These files contain reports on adverse drug events submitted by physicians, pharmacists, other healthcare professionals, manufacturers, and consumers from the U.S. and other countries. We built a database that integrated the quarterly report files using Oracle Database 11g software (Oracle, USA). The drugs selected for this investigation were omeprazole, esomeprazole, lansoprazole, dexlansoprazole, rabeprazole, pantoprazole, and ilaprazole. Before analyzing the data, a text-mining approach was utilized that stated the drugs in terms of their generic names and brand names. We obtained the brand names of each
PPI by querying the DrugBank database online. The DrugBank database is a comprehensive, freely accessible, online database containing information on drugs and drug targets (14). Then, we set the target drug as the primary suspect drug (PS) or second suspect drug (SS). We followed the FDA's recommendation to adopt the most recent and unique case number to identify duplicate reports of the same patient with different reporting sources and excluded these from the analysis. Then, a second process was performed using a record-linkage strategy, which groups records overlapping in three key fields: date the FDA received the first version of the case, age and sex of the patient and reporter country. Records with three overlaps were also considered duplicates.

\section{Definition of Hepatotoxicity Events}

This study relied on the definitions provided by MedDRA version 23.0 (15). To evaluate the effect of PPI treatment on HRAEs, different preferred terms (PTs) were identified with the Standardized MedDRA Query (SMQ) for "livery injury" and "acute hepatic failure" and the System Organ Class (SOC) for hepatobiliary disorder, and only reports that met both criteria were extracted. The number of selected PTs for "livery injury" and "acute hepatic failure" was 40 (see details in Supplementary Table S1).

Then, according to the main clinical features of adverse events, the selected PTs were divided into three types: "hepatocellular injury," "cholestasis," and "liver failure."

\section{Analysis}

All reported adverse events of interest were defined as "HRAE cases," and all reported other adverse events were defined as "noHRAE cases." To compare one of the PPI groups with the noPPI group, we calculated the RORs as (a: c)/ (b: d). RORs are expressed as point estimates with $95 \%$ confidence intervals (CIs). For signal detection, general qualitative judgments are viable, and whether a signal is detected or not depends on whether the signal indices exceed predefined thresholds: ROR values $>1$ and the number of total reports $\geq 3$ indicate potential exposure-event signals (16). Meanwhile, we defined the exposure-event signal of ROR value $\geq 5$ as the "strong signal."

This study analyzed the occurrences of HRAEs caused by PPIs and mainly included four aspects. First, the RORs of HRAEs caused by PPIs vs. non-PPI drugs were calculated. Second, we screened the "strong signal" adverse events in PPI-induced HRAEs. Third, the "strong signal" adverse events in HRAEs coowned by different PPIs were screened. Fourth, the RORs of "strong signal" adverse events in HRAEs co-owned by different PPIs were calculated.

We also analyzed the reports of the adverse events related to hepatic encephalopathy, which are more clinically concerning. We mainly mined the basic characteristics, clinical diagnosis and combined medication information of the patients in these reports.

Statistical significance was verified using chi-square tests. Data processing and analysis were conducted using SPSS version 25.0. Differences with $P$-values of $<0.05$ were considered statistically significant. 


\section{RESULTS}

After the exclusion of duplicates following the FDA recommendation, 8,221,278 reports in the FAERS database were of use. We used the generic name and brand name of the target drug to search the database, and the details are shown in Supplementary Table S2. There were 23,825 reports of PPIs considered as PS and SS, of which 3,253 reports were HRAEs. Because the adverse event reports of dexlansoprazole and ilaprazole were not found in the database, we omitted them in the final analysis. Thirty-one HRAEs were defined as exposureevent signals of the 40 caused by PPIs. The top five HRAE signals caused by PPIs were hepatitis cholestatic, cholestasis, fulminant hepatitis, subacute hepatic failure, and acute hepatitis, and the RORs were 13.777 (95\% CI 11.679-16.252), 12.939 (95\% CI 11.795-14.193), 8.315 (95\% CI 6.254-11.055), 7.301 (95\% CI 2.292-23.259), and 6.823 (95\% CI 5.560-8.373), respectively. The number of cases and RORs of HRAEs are detailed in Table 1.

We also summarized the signals of the HRAEs caused by each PPI (see details in Supplementary Tables S3-S7) and extracted 13 different signals for each PPI with ROR $\geq 5$ (see details in Figure 1). Among them, the signals of "hepatocellular injury" included acute hepatitis, hepatitis, liver injury, hepatic function abnormal and transaminases increased. The signals of "cholestasis" included cholestatic, hepatitis cholestatic, bilirubin conjugated increased and jaundice cholestatic. "Liver failure" included signals such as hepatitis fulminant, hepatic necrosis, coma hepatic, and hyperammonemia.

We found that the RORs of signals of "cholestasis" were generally higher than those of "hepatocellular injury." The signals of "liver failure" were mainly distributed in two or three PPIs. The simultaneous signals of more than four kinds of PPIs were cholestasis and hepatitis cholestatic. Pantoprazole had the most signal expression among the 13 signals, followed by lansoprazole. For the cholestasis signal, esomeprazole showed an ROR of 21.556 (95\% CI 17.592-26.413), which was higher than that of the other PPIs; Pantoprazole showed the highest ROR of 22.611 (95\% CI 17.794-28.733) in the hepatic cholestatic signal; Lansoprazole was the only PPI with expression in the coma hepatic signal, with an ROR of 10.424 (95\% CI 3.340-32.532); Rabeprazole and pantoprazole showed expression the hepatitis fulminant signal, with RORs of 40.240 (95\% CI 20.032-80.834) and 17.399 (95\% CI 12.017-25.191), respectively.

As shown in Figure 1, we found that the RORs of pantoprazole were higher in the signals of adverse events related to hepatic encephalopathy, therefore, pantoprazole was selected as the representative drug for subsequent information analysis. In the 28 reports of pantoprazole-related hepatitis encephalopathy, the proportion of men was higher than that of women, accounting for $\sim 68 \%$. The proportion of patients aged $\geq 60$ years was highest and was close to two-thirds. Reports of pantoprazole combined with more than three drugs were most frequent, accounting for $\sim 71 \%$. Antibiotics, antilipemic agents and contrast agents were commonly used drugs in combination with pantoprazole. The common clinical diagnosis when pantoprazole was used was skin infection (see details in Tables 2, 3 and Supplementary Table S8).
TABLE 1 | The number of cases and RORs of HRAE associated with all PPIs.

\begin{tabular}{|c|c|c|c|}
\hline PT & a & ROR (95\% two-sided Cl) & $P$ \\
\hline Acute hepatic failure & 103 & 3.262 (2.683-3.966) & $<0.001$ \\
\hline $\begin{array}{l}\text { Alanine } \\
\text { aminotransferase } \\
\text { abnormal* }^{*}\end{array}$ & 3 & $0.682(0.219-2.119)$ & 0.808 \\
\hline Ammonia increased* & 3 & $0.213(0.069-0.661)$ & 0.001 \\
\hline $\begin{array}{l}\text { Alanine } \\
\text { aminotransferase } \\
\text { increased }\end{array}$ & 249 & 2.052 (1.810-2.325) & $<0.001$ \\
\hline $\begin{array}{l}\text { Aspartate } \\
\text { aminotransferase } \\
\text { increased }\end{array}$ & 171 & $1.687(1.450-1.962)$ & $<0.001$ \\
\hline $\begin{array}{l}\text { Bilirubin conjugated } \\
\text { increased }\end{array}$ & 18 & $3.579(2.243-5.712)$ & $<0.001$ \\
\hline $\begin{array}{l}\text { Blood bilirubin } \\
\text { increased }\end{array}$ & 110 & 1.783 (1.477-2.152) & $<0.001$ \\
\hline Cholestasis & 491 & 12.939 (11.795-14.193) & $<0.001$ \\
\hline Coma hepatic & 5 & $2.826(1.166-6.845)$ & 0.035 \\
\hline $\begin{array}{l}\text { Hepatic } \\
\text { encephalopathy }\end{array}$ & 66 & 2.669 (2.092-3.405) & $<0.001$ \\
\hline $\begin{array}{l}\text { Hepatic enzyme } \\
\text { abnormal }^{\star}\end{array}$ & 13 & $1.036(0.600-1.787)$ & 0.887 \\
\hline $\begin{array}{l}\text { Hepatic enzyme } \\
\text { increased }\end{array}$ & 237 & 1.867 (1.642-2.123) & $<0.001$ \\
\hline Hepatic failure & 130 & 1.854 (1.559-2.205) & $<0.001$ \\
\hline $\begin{array}{l}\text { Hepatic function } \\
\text { abnormal }\end{array}$ & 187 & 2.416 (2.090-2.792) & $<0.001$ \\
\hline Hepatic necrosis & 33 & 4.957 (3.504-7.012) & $<0.001$ \\
\hline Hepatitis & 236 & $4.585(4.027-5.220)$ & $<0.001$ \\
\hline Hepatitis acute & 96 & 6.823 (5.560-8.373) & $<0.001$ \\
\hline Hepatitis cholestatic & 154 & 13.777 (11.679-16.252) & $<0.001$ \\
\hline Hepatitis fulminant & 50 & 8.315 (6.254-11.055) & $<0.001$ \\
\hline Hepatitis toxic & 16 & $2.775(1.693-4.551)$ & $<0.001$ \\
\hline Hepatotoxicity & 100 & $2.233(1.833-2.721)$ & $<0.001$ \\
\hline Hyperammonaemia & 22 & $1.828(1.201-2.784)$ & 0.009 \\
\hline Hyperbilirubinemia & 42 & 1.699 (1.253-2.303) & 0.001 \\
\hline Jaundice & 218 & 3.283 (2.870-3.755) & $<0.001$ \\
\hline Jaundice cholestatic & 31 & 3.926 (2.748-5.608) & $<0.001$ \\
\hline $\begin{array}{l}\text { Liver function test } \\
\text { abnormal }\end{array}$ & 160 & 2.251 (1.926-2.632) & $<0.001$ \\
\hline Liver injury & 122 & 3.337 (2.788-3.993) & $<0.001$ \\
\hline Liver transplant* & 3 & $0.332(0.107-1.032)$ & 0.043 \\
\hline $\begin{array}{l}\text { Subacute hepatic } \\
\text { failure }\end{array}$ & 3 & 7.301 (2.292-23.259) & 0.009 \\
\hline $\begin{array}{l}\text { Transaminases } \\
\text { abnormal }^{\star}\end{array}$ & 3 & $1.748(0.560-5.455)$ & 0.249 \\
\hline $\begin{array}{l}\text { Transaminases } \\
\text { increased }\end{array}$ & 170 & 3.074 (2.640-3.578) & $<0.001$ \\
\hline
\end{tabular}

HRAE, Hepatotoxicity-Related Adverse Effect; PPIs, Proton Pump Inhibitors; PT, Preferred Terms; " not defined signal of HRAE.

\section{DISCUSSION}

Abnormal liver enzymes (alanine aminotransferase, aspartate aminotransferase, alkaline phosphatase, etc.) are generally 


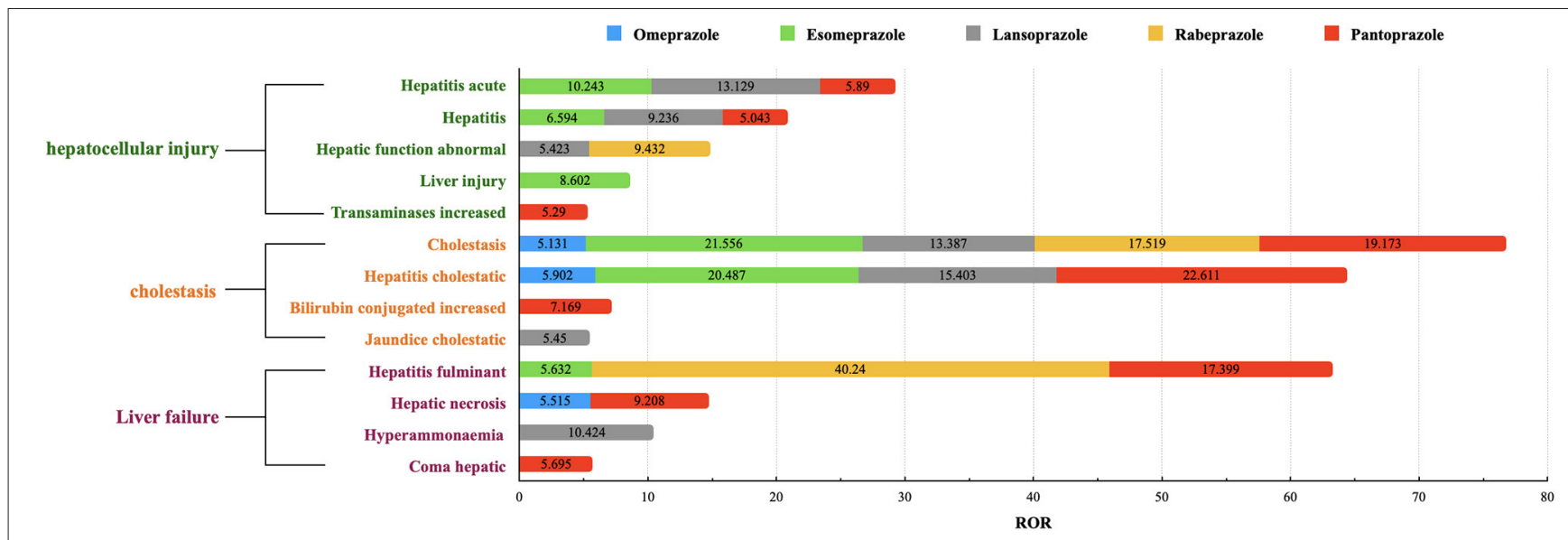

FIGURE 1 | The signals of RORs $\geq 5$ in each PPI. The ROR value shown in this figure is a ROR value of a HRAE signal caused by a single PPI. We extracted signals with $\mathrm{ROR} \geq 5$ for each PPI, and integrated the RORs of the same signal to get this figure.

TABLE 2 | The number of annual reports and clinical characteristics in Pantoprazole reports about hepatic encephalopathy.

\begin{tabular}{lc}
\hline Year & Number of reports $(\boldsymbol{n})$ \\
\hline 2013 & 0 \\
2014 & 0 \\
2015 & 3 \\
2016 & 0 \\
2017 & 10 \\
2018 & 14 \\
2019 & 1 \\
\hline Clinical characteristics & $\%(\boldsymbol{n})$ \\
\hline Sex & \\
Male & $19(67.86 \%)$ \\
Female & $6(21.43 \%)$ \\
Unknown & $3(10.71 \%)$ \\
Age (y, year) & \\
$<18$ & $0(0.00 \%)$ \\
$\geq 18$ and $<35$ & $2(7.14 \%)$ \\
$\geq 35$ and 60 & $3(10.71 \%)$ \\
$\geq 60$ & $19(67.86 \%)$ \\
Unknown & $4(14.29 \%)$ \\
\hline
\end{tabular}

regarded as common adverse events in clinical studies of PPIs or in the labels for the drug (17-20). In our study, although aspartate aminotransferase increased, liver function test abnormal and transaminases increased were defined as signaling adverse events, their ROR values were significantly different from those of cholestasis-associated signals (see details in Table 2). We think that there are several reasons for this result. First, mild liver enzyme elevation usually has fewer clinical manifestations and can only be diagnosed by relevant biochemical detection. Therefore, this situation results in a low report rate of liver enzyme abnormalities. Second, some reports tend to describe
TABLE 3 | Frequency of concomitant medication occurrences in Pantoprazole reports about hepatic encephalopathy.

\begin{tabular}{lc}
\hline Concomitant medication & Frequency of occurrence $\boldsymbol{n}(\%)$ \\
\hline Single drug & 0 \\
Combined 1 drug & 0 \\
Combined 2 drugs & $5(17.86 \%)$ \\
Combined 3 drugs & $3(10.71 \%)$ \\
Combined more than 3 drugs & $20(71.43 \%)$ \\
Total & 28 \\
\hline
\end{tabular}

clinical manifestations, such as hepatitis and liver injury, in which abnormal liver enzymes may coexist but were not reported.

Although abnormal liver enzymes may be underreported, there is no doubt that reports related to cholestasis have a stronger signal. In this study, we found that cholestatic signals were more likely to be detected both in all PPI HRAE reports and in individual PPI HRAE reports, which is not consistent with the information we obtained from the literature. Moreover, other signals related to "cholestasis" were reported at high rates. It may be that there were more obvious clinical manifestations in these patients or more severe symptoms, so cholestasis was therefore more widely reported.

We also screened the signals of each PPI, and the "strong signal" expression of each PPI was similar to that of all PPIs. Moreover, we also found that for the co-owned signals of the PPIs, only cholestasis was identified, suggesting that there was a significant difference in the "strong signal" between PPIs. Except for omeprazole, the other PPIs had high ROR values. This may be related to the fact that omeprazole was the first PPI to be used, and then influenced by Weber effect (21), the reports would decline year by year. Although it is not always observed (22). We also found that the RORs of the "cholestasis" signals were generally higher than those of "hepatocellular injury." This may be due to that "cholestasis" is more likely to cause doctors' 


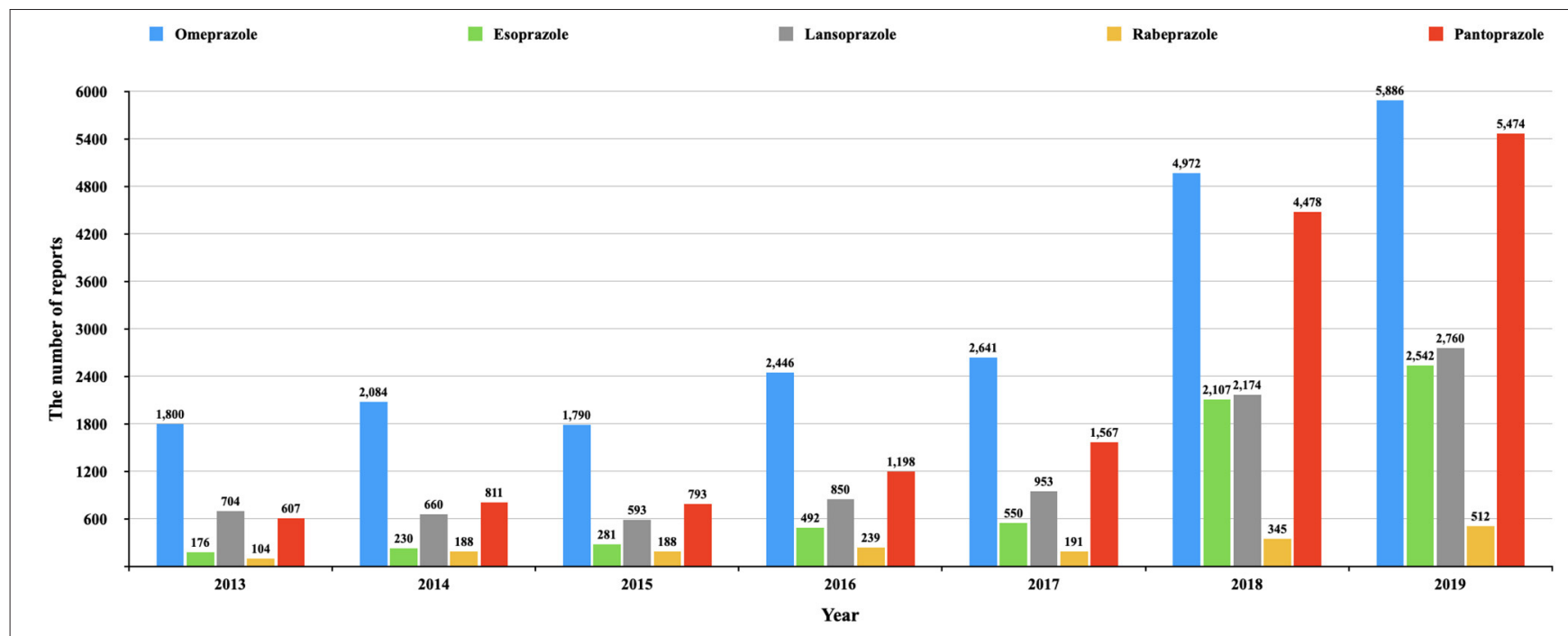

FIGURE 2 | The number of reports of each PPI in different year. This figure shows the number of reports of each PPI in different year (from the first quarter of 2013 to the fourth quarter of 2019).

influence than "hepatocellular injury." On the other hand, it could also be considered as a ripple effect (23).

In the single signal analysis, a "strong signal" of hepatitis fulminant was expressed in rabeprazole. Among all the PPIs, due to its late market time and small market scope, rabeprazole had the lowest number of reports, which were 1,797 , and the number of HRAE reports was 133. Since the ROR of hepatitis fulminant was very high ( $\mathrm{ROR}=40.240,95 \% \mathrm{CI} 20.032-80.834$ ), the authors believed that this could possibly be a false positive result because the data volume was small, which means that the frequency method could easily exaggerate the results and obtain many false positive results (16). The same reason was applied to the signal of coma hepatic. The ROR value of lansoprazole was high $(\mathrm{ROR}=10.424,95 \%$ CI 3.340-32.532), but only 3 cases were reported.

Omeprazole had the earliest clinical application and the most retrieved reports $(22,117)$, but the number of reports of HRAEs $(n=913)$ was lower than that of pantoprazole $(n=$ 1,134) (see details in Figure 2). The reported rates of adverse events in cholestasis and liver failure with pantoprazole were significantly higher than those with omeprazole. The signals of HARE in pantoprazole were the most common of all PPIs. This phenomenon also needs to be considered.

In recent years, more studies have revealed the relationship between hepatic encephalopathy and PPIs (24-26). In the above studies, the conclusions did not suggest which PPI had a higher risk of developing hepatic encephalopathy. Among all the signals about this adverse event, whether it was coma hepatic, hyperammonemia, or hepatic encephalopathy, there was also a higher reporting rate in a certain PPI. Among the PPIs with a high reporting rate in these signals, we selected pantoprazole as the representative drug to analyze the characteristics of the reports of hepatic encephalopathy. We found that most reports occurred in the past 3 years, consistent with the time when physicians were aware of such adverse events. In terms of the clinical characteristics of the patients, males aged over 65 years had the highest proportion in the reports (see details in Table 3). In a recent meta-analysis on the use of Proton pump inhibitor therapy and hepatic encephalopathy risk in cirrhotic patients, similar results were obtained, the proportion of male patients was higher than that of women, and most of them were middle-aged and elderly patients (27). This may be related to the epidemiological characteristics of patients with liver disease. Generally, patients with liver disease have a higher incidence of hepatic encephalopathy (28), and the proportion of male patients with liver disease was often higher than that of female patients $(29,30)$. However, in patients without liver diseases, the reports of hepatic encephalopathy caused by PPIs are rare (31), it is difficult to determine the gender differences. Furthermore, due to the limitations of the study (32), the reported rate of adverse events cannot be equated to the actual incidence of adverse events. Therefore, even though the proportion of male is higher in the report, further clinical studies are also needed to further clarify the relationship between gender and hepatic encephalopathy caused by PPIs. In addition, another clinical study found that the use of different PPI, liver cirrhosis patients with a slightly different risk of hepatic encephalopathy, in which the risk of pantoprazole was higher, and OR value was 2.05 (25).

We also tried to explore the clinical diagnosis of patients through the description of indications in the reports, but unfortunately, due to the defects of the reports themselves, many indications for drug use were not accurately described. The indications for some of the drugs that were obtained did not accurately reflect the extent to which underlying diseases contribute to adverse events (see details in Supplementary Table S8).

We described the situation of the combined use of drugs in the reports and found that $71.43 \%$ of the reports, more than three drugs were used at the same time (see details in Table 3). We also extracted information on drugs with a high frequency 
of combined use, which were mainly antibiotics, but did not find a clear causal relationship with the occurrence of adverse events (see details in Supplementary Table S8). Therefore, the information collected in the reports was similar to the results of clinical studies.

From a statistical point of view, adverse event signal strength can indicate the magnitude of the correlation between drugs and events. Therefore, it is currently believed that signal detection can preliminarily describe the possible relationship between drugs and adverse events and aid in further evaluation and research. At present, the only method that can be used for adverse event signal detection is the disproportionality method, including the proportional reporting ratio (PRR) method, ROR method, Bayesian method, etc. The ROR method was chosen because it is more sensitive and accurate than other methods (33). Considering that the main purpose of our study was to detect the signals of HRAE of PPIs, we did not continue to verify the results of the other methods in our study. This is also a limitation of the statistical results of the study.

This study has limitations as well. First, while the database has the merit of providing an early real-world perspective, the quality of the diagnosis of adverse events likely widely varies, and our study captures a short time frame early in the medication market life. And the study was a retrospective study, which only interpreted the reported data from the first quarter of 2013 to the fourth quarter of 2019. As a result, based on the data obtained from this period, the statistical results may have some limitations. Second, there is limited information in FAERS regarding a wide range of patient health characteristics, thus limiting the ability to control confounding effects (34). For example the association of a drug with an adverse event might be explained by those of other drugs which are often co-administered. For another example, PPIs are metabolized by CYP2C19; and so, the hepatotoxicity-related adverse effects could be affected by CYP2C19 genotype. Indeedly, the drug-drug interaction is subjected to CYP2C19 genotype (35). Of course, FAERS database is not included genotyping information. Third, since the reports of adverse events in FAERS are voluntary, it is difficult to complete the quality evaluation of the reports. Sometimes, the basic diseases of patients will also have adverse manifestations of liver and gallbladder $(36,37)$. Therefore, we retrieved the reported data of major adverse events with high ROR value of pantoprazole, and sorted out the indications of the top 10 of each HRAE to judge the patient's disease background. Unfortunately, we found that the main indication in the report was "PRODUCT USED FOR UNKNOWN INDICATION," so it is difficult to distinguish whether the occurrence of adverse events is related to the underlying disease

\section{REFERENCES}

1. Maes ML, Fixen DR, Linnebur SA. Adverse effects of protonpump inhibitor use in older adults: a review of the evidence. Ther Adv Drug Saf. (2017) 8:273-97. doi: 10.1177/2042098617 715381

2. Strand DS, Kim D, Peura DA. 25 Years of proton pump inhibitors: a comprehensive review. Gut Liver. (2017) 11:27-37. doi: 10.5009/gnl15502 (see details in Supplementary Table S9). Fourth, adverse events are underreported in spontaneous reporting systems in general $(38,39)$. The rate of reporting can vary with the particular adverse event (40), but averages just 6\% (38). Even though the reporting rate has dramatically improved, the FAERS database is still not appropriate for estimating incidence rates, due to the absence of a denominator (32). After considering causality restraints of the current analysis, it is recommended that robust epidemiological studies should be conducted to further validate the hypothesis to draw conclusions that contribute to clinical practice.

\section{CONCLUSION}

This study found that the "strong signal" of HRAEs in PPIs were cholestasis and hepatitis cholestatic, whether in all the PPI HRAE reports or within a single species of PPI HRAE reports. Rabeprazole and pantoprazole were more likely to cause hepatitis fulminant than the other PPIs. By analyzing the reports of pantoprazole-induced hepatic encephalopathy, we found that patients aged over 65 years and males reported the highest rate, which was similar to the results of known clinical studies. However, from the combination of drugs and indications of drugs, no positive and significant results were obtained. Owing to the limitations of the study, further studies are needed to identify the effects of other combinations of drugs on PPIinduced HRAEs.

\section{DATA AVAILABILITY STATEMENT}

Publicly available datasets were analyzed in this study. This data can be found at: https://fis.fda.gov/extensions/FPD-QDEFAERS/FPD-QDE-FAERS.html.

\section{AUTHOR CONTRIBUTIONS}

YZ and YD contributed to the data analysis and overall construction of the paper. XY contributed to the management of adverse events. ZZ peer reviewed the paper internally. DS conceived the paper and had overall oversight of the data analysis and paper construction. All authors contributed to the article and approved the submitted version.

\section{SUPPLEMENTARY MATERIAL}

The Supplementary Material for this article can be found online at: https://www.frontiersin.org/articles/10.3389/fmed. 2021.648164/full\#supplementary-material

3. Schoenfeld AJ, Grady D. Adverse effects associated with proton pump inhibitors. JAMA Intern Med. (2016) 176:172-4. doi: 10.1001/jamainternmed.2015.7927

4. Aslan M, Celik Y, Karadas S, Olmez S, Cifci A. Liver hepatotoxicity associated with pantoprazole: a rare case report. Wien Klin Wochenschr. (2014) 126:3902. doi: 10.1007/s00508-014-0535-3

5. El-Matary W, Dalzell M. Omeprazole-induced hepatitis. Pediatr Emerg Care. (2005) 21:529-30. doi: 10.1097/01.pec.0000173350.45648.9b 
6. Christe C, Stoller R, Vogt N. Omeprazole-induced hepatotoxicity? a case report. Pharmacoepidemiol Drug Saf. (1998) 7(Suppl. 1):S41-4. doi: 10.1002/ (SICI)1099-1557(199808)7:1+ <S41::AID-PDS347>3.0.CO;2-2

7. VigiAccess ${ }^{\mathrm{TM}}$. Uppsala Monitoring Center. Available online at: http://www. vigiaccess.org/ (accessed May 16, 2020).

8. Ji HH, Tang XW, Dong Z, Song L, Jia YT. Adverse event profiles of antiCTLA-4 and Anti-PD-1 monoclonal antibodies alone or in combination: analysis of spontaneous reports submitted to FAERS. Clin Drug Investig. (2019) 39:319-30. doi: 10.1007/s40261-018-0735-0

9. Serebruany VL, Hall TS, Atar D, Agewall S, Hyun Kim M, Geudelin B, et al. Mortality and adverse events with brand and generic clopidogrel in the US food and drug administration adverse event reporting system. Eur Heart J Cardiovasc Pharmacother. (2019) 5:210-5. doi: 10.1093/ehjcvp/pvy035

10. Szarfman A, Machado SG, O'Neill RT. Use of screening algorithms and computer systems to efficiently signal higher-than-expected combinations of drugs and events in the US FDA's spontaneous reports database. Drug Saf. (2002) 25:381-92. doi: 10.2165/00002018-200225060-00001

11. Almenoff J, Tonning JM, Gould AL, Szarfman A, Hauben M, Ouellet-Hellstrom R, et al. Perspectives on the use of data mining in pharmaco-vigilance. Drug Saf. (2005) 28:981-1007. doi: 10.2165/00002018-200528110-00002

12. van Puijenbroek EP, Bate A, Leufkens HG, Lindquist $M$, Orre R, Egberts AC. A comparison of measures of disproportionality for signal detection in spontaneous reporting systems for adverse drug reactions. Pharmacoepidemiol Drug Saf. (2002) 11:3-10. doi: 10.1002/pds.668

13. US Food and Drug Administration. Adverse Event Reporting System (AERS). Available online at: http://www.fda.gov/Drugs/GuidanceCompliance/ RegulatoryInformation/Surveillance/AdverseDrugEffects/default.htm (accessed June 15, 2020).

14. DrugBank Database. Available online at: http://www.drugbank.ca/ (accessed June 06, 2020).

15. Maintenance and Support Services Organization. Medical Dictionary for Regulatory Activities. Available online at: http://www.meddramsso.com/ (accessed July 03, 2020).

16. Bate A, Evans SJ. Quantitative signal detection using spontaneous ADR reporting. Pharmacoepidemiol Drug Saf. (2009) 18:427-36. doi: $10.1002 /$ pds. 1742

17. Sugano K, Kinoshita Y, Miwa H, Takeuchi T, Esomeprazole NSAID Preventive Study Group. Safety and efficacy of long-term esomeprazole $20 \mathrm{mg}$ in Japanese patients with a history of peptic ulcer receiving daily non-steroidal anti-inflammatory drugs. BMC Gastroenterol. (2013) 13:54. doi: 10.1186/1471-230X-13-54

18. Sugano K, Kinoshita Y, Miwa H, Takeuchi T, Esomeprazole NSAID Preventive Study Group. Randomised clinical trial: esomeprazole for the prevention of nonsteroidal anti-inflammatory drug-related peptic ulcers in Japanese patients. Aliment Pharmacol Ther. (2012) 36:115-25. doi: 10.1111/j.1365-2036.2012.05133.x

19. Mizokami Y, Oda K, Funao N, Nishimura A, Soen S, Kawai T, et al. Vonoprazan prevents ulcer recurrence during long-term NSAID therapy: randomised, lansoprazole-controlled non-inferiority and single-blind extension study. Gut. (2018) 67:1042-51. doi: 10.1136/gutjnl-2017-314010

20. Labeling-Package Insert for OMEPRAZOLE SODIUM Tablet, Delayed Release. Teva Pharmaceuticals USA, Inc. (2017).

21. Hartnell NR, Wilson JP. Replication of the Weber effect using postmarketing adverse event reports voluntarily submitted to the United States food and drug administration. Pharmacotherapy. (2004) 24:743-9. doi: $10.1592 /$ phco.24.8.743.36068

22. McAdams MA, Governale LA, Swartz L, Hammad TA, Dal Pan GJ. Identifying patterns of adverse event reporting for four members of the angiotensin II receptor blockers class of drugs: revisiting the Weber effect. Pharmacoepidemiol Drug Saf. (2008) 17:882-9. doi: 10.1002/pds.1633

23. Pariente A, Gregoire F, Fourrier-Reglat A, Haramburu F, Moore N. Impact of safety alerts on measures of disproportionality in spontaneous reporting databases: the notoriety bias. Drug Saf. (2007) 30:891-8. doi: 10.2165/00002018-200730100-00007

24. O'Leary JG, Reddy KR, Wong F, Kamath PS, Patton HM, Biggins SW, et al. Long-term use of antibiotics and proton pump inhibitors predict development of infections in patients with cirrhosis. Clin Gastroenterol Hepatol. (2015) 13:753-9.e1-2. doi: 10.1016/j.cgh.2014.07.060
25. Tsai CF, Chen MH, Wang YP, Chu CJ, Huang YH, Lin HC, et al. Proton pump inhibitors increase risk for hepatic encephalopathy in patients with cirrhosis in a population study. Gastroenterology. (2017) 152:134-41. doi: 10.1053/j.gastro.2016.09.007

26. Nardelli S, Gioia S, Ridola L, Farcomeni A, Merli M, Riggio O. Proton pump inhibitors are associated with minimal and overt hepatic encephalopathy and increased mortality in patients with cirrhosis. Hepatology. (2019) 70:640-9. doi: 10.1002/hep.30304

27. Shi D, Zhou Z, Dai Y, Pan X, Cao Q. Proton pump inhibitor therapy and hepatic encephalopathy risk in cirrhotic patients: a systematic review with meta-analysis. Clin Drug Investig. (2019) 39:847-56. doi: 10.1007/s40261-019-00810-8

28. Wijdicks EF. Hepatic encephalopathy. N Engl J Med. (2016) 375:1660-70. doi: 10.1056/NEJMra1600561

29. Asrani SK, Hall L, Hagan M, Sharma S, Yeramaneni S, Trotter J, et al. Trends in chronic liver disease-related hospitalizations: a population-based study. Am J Gastroenterol. (2019) 114:98-106. doi: 10.1038/s41395-018-0365-4

30. Hashimoto E, Tokushige K. Prevalence, gender, ethnic variations, and prognosis of NASH. J Gastroenterol. (2011) 46(Suppl. 1):63-9. doi: 10.1007/s00535-010-0311-8

31. Jochem V, Kirkpatrick R, Greenson J, Brogan M, Sturgis T, Cook-Glenn C. Fulminant hepatic failure related to omeprazole. Am J Gastroenterol. (1992) 87:523-5.

32. Rodriguez EM, Staffa JA, Graham DJ. The role of databases in drug postmarketing surveillance. Pharmacoepidemiol Drug Saf. (2001) 10:407-10. doi: $10.1002 /$ pds.615

33. Rothman KJ, Lanes S, Sacks ST. The reporting odds ratio and its advantages over the proportional reporting ratio. Pharmacoepidemiol Drug Saf. (2004) 13:519-23. doi: 10.1002/pds. 1001

34. Stephenson WP, Hauben M. Data mining for signals in spontaneous reporting databases: proceed with caution. Pharmacoepidemiol Drug Saf. (2007) 16:35965. doi: $10.1002 /$ pds. 1323

35. Sakaeda T, Tamon A, Kadoyama K, Okuno Y. Data mining of the public version of the FDA adverse event reporting system. Int J Med Sci. (2013) 10:796-803. doi: 10.7150/ijms.6048

36. Granito A, Muratori P, Muratori L, Pappas G, Cassani F, Worthington $\mathrm{J}$, et al. Antinuclear antibodies giving the 'multiple nuclear dots' or the 'rim-like/membranous' patterns: diagnostic accuracy for primary biliary cirrhosis. Aliment Pharmacol Ther. (2006) 24:1575-83. doi: 10.1111/j.1365-2036.2006.03172.x

37. Granito A, Muratori P, Quarneti C, Pappas G, Cicola R, Muratori L. Antinuclear antibodies as ancillary markers in primary biliary cirrhosis. Expert Rev Mol Diagn. (2012) 12:65-74. doi: 10.1586/erm.11.82

38. Hosohata K, Masuda S, Katsura T, Takada Y, Kaido T, Ogura Y, et al. Impact of intestinal CYP2C19 genotypes on the interaction between tacrolimus and omeprazole, but not lansoprazole, in adult living-donor liver transplant patients. Drug Metab Dispos. (2009) 37:821-6. doi: 10.1124/dmd.108.025833

39. Hazell L, Shakir SA. Under-reporting of adverse drug reactions: a systematic review. Drug Saf. (2006) 29:385-96. doi: 10.2165/00002018-200629050-00003

40. Lopez-Gonzalez E, Herdeiro MT, Figueiras A. Determinants of underreporting of adverse drug reactions: a systematic review. Drug Saf. (2009) 32:19-31. doi: 10.2165/00002018-200932010-00002

Conflict of Interest: The authors declare that the research was conducted in the absence of any commercial or financial relationships that could be construed as a potential conflict of interest.

Publisher's Note: All claims expressed in this article are solely those of the authors and do not necessarily represent those of their affiliated organizations, or those of the publisher, the editors and the reviewers. Any product that may be evaluated in this article, or claim that may be made by its manufacturer, is not guaranteed or endorsed by the publisher.

Copyright (c) 2021 Zeng, Dai, Zhou, Yu and Shi. This is an open-access article distributed under the terms of the Creative Commons Attribution License (CC BY). The use, distribution or reproduction in other forums is permitted, provided the original author(s) and the copyright owner(s) are credited and that the original publication in this journal is cited, in accordance with accepted academic practice. No use, distribution or reproduction is permitted which does not comply with these terms. 